\title{
Sales segmentation for a mobile phone service through logistic regression algorithm
}

\author{
Autores \\ Amelec Viloria, Guojun Wang, Mercedes Gaitan
}

\begin{abstract}
The research aims to describe the CRISP-DM method to identify optimal customer groups that are likely to migrate from a prepaid to postpaid plan in order to formulate an improvement plan in call management by sorting the database. The logistic regression model was applied to analyze the characteristics generated by the purchase of different services. In this sense, groups differentiated by their probability of sales success (migrating from a prepaid to postpaid plan) were found, as segments that reflect needs and characteristics that allow to design marketing actions focused on the objective of increasing the effectiveness, contactability, and sales.

Palabras clave

Call center, CRISP-DM, Logistic regression model.
\end{abstract}

